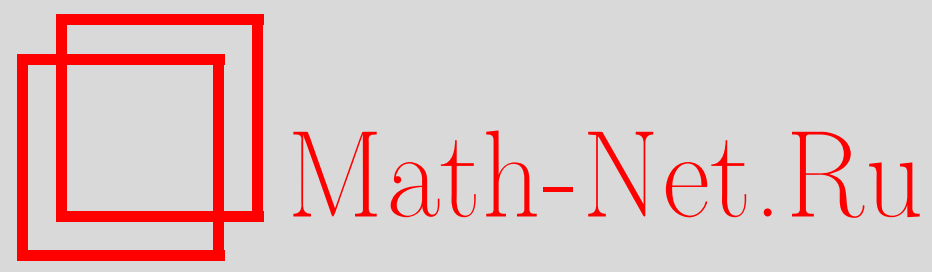

А. С. Кочуров, Аппроксимация кусочно-постоянными функциями на квадрате, Матем. заметки, 2004, том 75, выпуск 4, 592-602

DOI: https://doi.org/10.4213/mzm54

Использование Общероссийского математического портала Math-Net.Ru подразумевает, что вы прочитали и согласны с пользовательским соглашением http://www.mathnet.ru/rus/agreement

Параметры загрузки:

IP : 3.93.64.190

26 апреля 2023 г., 02:19:13 


\section{АППРОКСИМАЦИЯ КУСОЧНО-ПОСТОЯННЫМИ ФУНКЦИЯМИ НА КВАДРАТЕ}

\section{А. С. Кочуров}

В этой работе рассматриваются некоторые алгоритмы аппроксимации функций, определенных на единичном квадрате $\mathbf{I}=[0,1]^{2}$ из $\mathbb{R}^{2}$. В качестве аппарата приближения используются функции типа сплайнов Лагранжа нулевого порядка. Их отличие от стандартных лагранжевых сплайнов на плоскости заключено в правиле выбора линий сетки, по которой предполагается строить сплайн: вместо семейства параллельных прямых, задающих узлы интерполяции, используется набор одномерных сплайнов.

Библиография: 1 название.

В [1] был предложен метод аппроксимации функций двух переменных кусочно-постоянными функциями. Для него были введены некоторые характеристики гладких функций, в терминах которых оказалось возможным получать точные по порядку оценки скорости такой аппроксимации. Попытки распространить метод и характеристики на более общие функциональные классы привели к появлению этой работы. Как представляется, главньм в ней является метод приближения функций двух переменных, решающий обратную задачу: по заданной точности приближения построить аппроксимирующий агрегат. В то же время определенное внимание уделяется и прямой задаче-по заданным параметрам агрегата получить оценку точности, с которой можно осуществить приближение. Для этого сделано “обобщение" некоторых характеристик, введенных в [1], и в их терминах получены оценки сверху для скорости аппроксимации. Вопрос об их точности (т.е. оценки снизу), в отличие от [1], не затрагивается.

Всюду ниже будем обозначать через $z$ с индексом и без него точку из $\mathbf{I}=[0,1]^{2}$, через $x$ и $y$ - координаты $z$. Пусть функция $f(\cdot) \in C(\mathbf{I})$ при любом $x \in[0,1]$ имеет ограниченную по $y$ вариацию: $f(x, \cdot) \in \operatorname{Var}_{0}^{1}$ (под $\operatorname{Var}_{0}^{y}, y \in(0,1]$, будем понимать класс функций $g$, для которых

$$
\operatorname{Var}_{0}^{y}(g)=\sup _{\substack{v(\cdot) \in C_{0}^{1}(0, y) \\\|v\|_{C} \leqslant 1}} \int_{0}^{y} g(s) \frac{d v}{d s} d s<\infty
$$

здесь $C_{0}^{1}(0, y)$ - класс непрерьвно дифференцируемых функций с носителем внутри $\left.(0, y)\right)$;

Работа выполнена при финансовой поддержке Российского фонда фундаментальных исследований, грант № 97-01-01007. 
пусть $\varepsilon_{1}>0$ - фиксированное число и функция

$$
\Lambda(x, y)=\operatorname{Var}_{0}^{y} f(x, \cdot)+\varepsilon_{1} \cdot y, \quad(x, y) \in \mathbf{I}, \quad y>0, \quad \Lambda(x, 0)=0, \quad x \in[0,1]
$$

непрерьвна на I (в этом определении $\varepsilon_{1}$ вводится для обеспечения строгой монотонности $\Lambda(x, y)$ по переменной $y$; оно может быть выбрано сколь угодно малым). Дальнейшие построения в значительной, по мнению автора, степени продиктованы правилами приближения функций одного переменного сплайнами. Положим

$$
f(x, y)=: \Lambda(x, y)-\mathscr{M}(x, y)+f(x, 0) .
$$

Хорошо известно, что функции $\Lambda(x, \cdot), \mathscr{M}(x, \cdot)$ строго возрастают при любом фиксированном $x \in[0,1]$. Приблизим отдельно каждую из $\Lambda(\cdot), \mathscr{M}(\cdot), f(\cdot, 0)$. Начнем с функции $\Lambda(\cdot)$. Обозначим через $h(x, \cdot), x \in[0,1]$, функцию, обратную к $\Lambda(x, \cdot)$. Пусть $H \subset[0,1] \times[0, \lambda]-$ область определения $h(\cdot), G=\operatorname{int} H-$ внутренность $H$ (здесь $[0, \lambda]$ - образ I при отображении $\Lambda(\cdot))$; пусть $I(\Lambda)=\operatorname{Var}_{0}^{1}(\Lambda(\cdot, 1))$.

ОПРЕДЕЛЕНИЕ 1. Пусть $\mathscr{P}_{r}, r \in \mathbb{Z}_{+},-$класс полиномов степени не вьше $r, n \in \mathbb{N}$; $s(\cdot): \Delta \rightarrow[0,1]-$ кусочно-полиномиальная функция, определенная на отрезке $\Delta \subseteq[0,1]$, такая, что для некоторого разбиения $a_{0} \leqslant a_{1} \leqslant \cdots \leqslant a_{n}$ отрезка $\left[a_{0}, a_{n}\right]=\Delta$

$$
s(\cdot)=p_{i}(\cdot) \in \mathscr{P}_{r}
$$

на любом из интервалов $\left(a_{i}, a_{i+1}\right), i=0,1, \ldots, n-1 ;$ пусть $S_{n}^{r}[0,1]-$ класс кусочно-полиномиальных функций типа $s(\cdot)$, которые начинаются и заканчиваются на гранище квадрата $\mathbf{I}$, т.е. либо $a_{0}=0$, либо $s\left(a_{0}\right)=0$ или $s\left(a_{0}\right)=1$ и, аналогично, для $a_{n}$ : либо $a_{n}=1$, либо $s\left(a_{n}\right)=0$ или $s\left(a_{n}\right)=1$. Для функции $s(\cdot) \in S_{n}^{r}[0,1]$ через $\bar{s}$ обозначим ее обобщенный график

$$
\begin{aligned}
\bar{s}=\{(x, s(x)) \mid x \in \Delta\} \cup\left\{\left(a_{0}, y\right) \mid y \in\left[s\left(a_{0}\right), p_{0}\left(a_{0}\right)\right]\right\} \cup \\
\cup\left\{\left(a_{n}, y\right) \mid y \in\left[s\left(a_{n}\right), p_{n-1}\left(a_{n}\right)\right]\right\} \bigcup_{i=1}^{n-1}\left\{\left(a_{i}, y\right) \mid y \in\left[p_{i-1}\left(a_{i}\right), p_{i}\left(a_{i}\right)\right]\right\} .
\end{aligned}
$$

ОПРЕДЕЛЕНИЕ 2. Пусть $S_{n}^{r}, r \in \mathbb{Z}_{+}, n \in \mathbb{N},-$ класс кусочно-постоянных функций $S(z)$, определенных на $\mathbf{I}$, для которых при некотором наборе $n_{j} \in \mathbb{N}, j \in J, \sum_{j \in J} n_{j} \leqslant n$, выполняются следуюшие условия (положим $z=(x, y)$ ):

1) при каждом $x \in[0,1]$ функция $S(x, \cdot)$ не убывает по переменной $y$;

2 ) множество $\bar{S}$, состоящее из объединения всех гранищ областей постоянства $S(\cdot)$, может быть представлено в виде $\bigcup\left\{\bar{s}_{j} \mid j \in J\right\}$ так, что каждое из $\bar{s}_{j}$ - обобщенньй график функции $s_{j}(\cdot) \in S_{n_{j}}^{r}[0,1], j \in J$;

3) значение $S\left(z_{0}\right)$ для $z_{0} \in \bar{S}$ равно одному из предельных значений, которые принимает $S(z)$, когда $z$ приближается к $z_{0}$ и остается вне $\bar{S}$. 
Через $\|\Lambda\|_{B}$ обозначим норму $\sup _{z \in \mathbf{I}}|\Lambda(z)|$ в пространстве ограниченных функций, через $E_{n}^{r}(\Lambda)$ - величину $\inf _{S \in S_{n}^{r}}\|\Lambda-S\|_{B}$.

Пусть $w \in(0, \lambda), G \cap\{(0,1) \times w\}=\bigcup_{j}\left(x_{j, 1}, x_{j, 2}\right) \times w-$ разложение открытого в $(0,1) \times w$ множества на не более чем счетное число максимальных связных компонент. Определим отображение $\Omega^{\star}(w)$ одним из двух способов:

а) в первом случае обозначим через $p\left(\cdot, \tau_{1}, \tau_{2}, w\right), e\left(\tau_{1}, \tau_{2}, w\right)$ соответственно полином и величину “наименьшего $w$-уклонения" на $\left[\tau_{1}, \tau_{2}\right]$ :

$$
\begin{aligned}
& p\left(\cdot, \tau_{1}, \tau_{2}, w\right) \\
& \quad=\arg \left\{e\left(\tau_{1}, \tau_{2}, w\right)=\inf \left\{\max _{t \in\left[\tau_{1}, \tau_{2}\right]}|w-\Lambda(t, p(t))| \mid p \in \mathscr{P}_{r}, p(\cdot):\left[\tau_{1}, \tau_{2}\right] \rightarrow[0,1]\right\}\right\}
\end{aligned}
$$

б) во втором случае введем дополнительное ограничение гладкости: $h(\cdot) \in C^{r}(H)$ и

$$
\begin{gathered}
p\left(\cdot, \tau_{1}, \tau_{2}, w\right)=p\left(\cdot, \tau_{1}, w\right)=\sum_{i=0}^{r} \frac{h_{x}^{(i)}\left(\tau_{1}, w\right)\left(\cdot-\tau_{1}\right)^{i}}{i !} \\
e\left(\tau_{1}, \tau_{2}, w\right)=\left|w-\Lambda\left(\tau_{2}, p\left(\tau_{2}, \tau_{1}, w\right)\right)\right|
\end{gathered}
$$

если $p\left(t, \tau_{1}, w\right) \in[0,1]$ при всех $t \in\left[\tau_{1}, \tau_{2}\right]$, если нет - считаем $e\left(\tau_{1}, \tau_{2}, w\right)=0$. В любом случае пусть

$$
\Omega^{\star}(w)=\sum_{j} \sup _{x_{j, 1} \leqslant \tau_{1}<\tau_{2}<\cdots<\tau_{m} \leqslant x_{j, 2}} \sum_{k=1}^{m-1} e\left(\tau_{k}, \tau_{k+1}, w\right)^{1 /(r+1)} .
$$

Чтобы не касаться вопросов, связанных с измеримостью функции $\Omega^{\star}(\cdot)$, рассмотрим

$$
\Omega\left(w_{0}\right)=\liminf _{w \rightarrow w_{0}} \Omega^{\star}(w), \quad w_{0} \in(0, \lambda), \quad I(\Lambda, r)=\int_{(0, \lambda)} \Omega(w) d w .
$$

ЗАмЕчАниЕ 1 . В качестве правила выбора полинома $p\left(\cdot, \tau_{1}, \tau_{2}, w\right)$ в этом определении можно выбирать аналог любой “естественной” процедуры интерполящии функции $h(\cdot, w)$, используемой при аппроксимации функций сплайнами на $[0,1]$.

ЗАмЕчАниЕ 2. В этом замечании будем считать, что функции $\Lambda(\cdot)$ и $h(\cdot)$ достаточно гладкие. Для каждого $j$, участвуюшего в определении $\Omega^{\star}(w)$, рассмотрим сумму

$$
\sum_{k=1}^{m} e\left(\tau_{k}, \tau_{k+1}, w\right)^{1 /(r+1)}, \quad \tau_{k}=x_{j, 1}+\left(x_{j, 2}-x_{j, 1}\right) \frac{k-1}{m}, \quad k=1, \ldots, m+1 .
$$

Вычислим предел, к которому она стремится с ростом $m$ :

$$
\begin{aligned}
\sum_{k=1}^{m} e\left(\tau_{k}, \tau_{k+1}, w\right)^{1 /(r+1)} & =\left(\sum_{k=1}^{m}\right)^{\prime}\left|\Lambda\left(\tau_{k}^{\prime}, h\left(\tau_{k}^{\prime}, w\right)\right)-\Lambda\left(\tau_{k}^{\prime}, p\left(\tau_{k}^{\prime}, \tau_{k}, \tau_{k+1}, w\right)\right)\right|^{1 /(r+1)} \\
& =\left(\sum_{k=1}^{m}\right)^{\prime}\left|\Lambda_{y}\left(\tau_{k}^{\prime}, \xi_{k}\right)\left(h\left(\tau_{k}^{\prime}, w\right)-p\left(\tau_{k}^{\prime}, \tau_{k}, \tau_{k+1}, w\right)\right)\right|^{1 /(r+1)} \\
& \rightarrow \int_{x_{j, 1}}^{x_{j, 2}}\left|\Lambda_{y}(\tau, h(\tau, w)) \cdot h_{x}^{(r+1)}(\tau, w) /(r+1) !\right|^{1 /(r+1)} d \tau
\end{aligned}
$$


(здесь $\tau_{k}^{\prime} \in\left[\tau_{k}, \tau_{k+1}\right]$ - промежуточная точка в случае (a), $\tau_{k}^{\prime}=\tau_{k+1}$ - в случае (б), $\xi_{k} \in\left[h\left(\tau_{k}^{\prime}, w\right), p\left(\tau_{k}^{\prime}, \tau_{k}, \tau_{k+1}, w\right)\right]-$ промежуточная точка, $\left(\sum_{k=1}^{m}\right)^{\prime}$ означает неполное суммирование: некоторые слагаемые, согласно определению $e\left(\tau_{1}, \tau_{2}, w\right)$, могут быть нулевыми, однако вклад их с ростом $m$ стремится к нулю).

Поэтому в определенном смысле величину $I(\Lambda, r)$ можно считать "обобщенным" аналогом для

$$
\bar{I}(\Lambda, r)=\int_{(0, \lambda)} \sum_{j} \int_{x_{j, 1}}^{x_{j, 2}}\left|\frac{\Lambda_{y}(\tau, h(\tau, w)) \cdot h_{x}^{(r+1)}(\tau, w)}{(r+1) !}\right|^{1 /(r+1)} d \tau d w .
$$

Сделав замену переменной $w \rightarrow y, y=h(\tau, w)$, для каждого фиксированного $\tau$ получим (см. [1]):

при $r=0$

$$
\bar{I}(\Lambda, 0)=\int_{\mathbf{I}}\left|\Lambda_{x} \Lambda_{y}\right| d x d y
$$

при $r=1$

$$
\bar{I}(\Lambda, 1)=\int_{\mathbf{I}}\left|\frac{1}{2}\left(\Lambda_{x x}\left(\Lambda_{y}\right)^{2}-2 \Lambda_{x y} \Lambda_{x} \Lambda_{y}+\Lambda_{y y}\left(\Lambda_{x}\right)^{2}\right)\right|^{1 / 2} d x d y .
$$

\section{Введем}

$d=\{z=(x, 1) \mid x \in[0,1]\}, \quad d_{i}=\{z=(i, y) \mid y \in[0,1]\}, \quad i=0,1, \quad d_{2}=d \cup d_{0} \cup d_{1}$.

Упорядочим точки $z \in d_{2}$ по часовой стрелке: считаем, что $z_{1} \leqslant z_{2}, z_{1}, z_{2} \in d_{2}$, если либо $z_{1}=\left(x_{1}, y_{1}\right) \in d_{0}$ и $z_{2} \in d \cup d_{1}$ или $z_{2}=\left(x_{2}, y_{2}\right) \in d_{0}$ и $y_{1} \leqslant y_{2}$; либо $z_{1} \in d$ и $z_{2} \in d_{1}$ или $z_{2} \in d$ и $x_{1} \leqslant x_{2}$; либо $z_{1}, z_{2} \in d_{1}, y_{1} \geqslant y_{2}$.

ТЕОРема. В сделанных предположениях относительно $n, r$ u $\Lambda(\cdot)$

$$
E_{n}^{r}(\Lambda) \leqslant 4 \max \left\{\left(\frac{I(\Lambda, r)}{n}\right)^{(r+1) /(r+2)}, \quad \frac{2(I(\Lambda)+\Lambda(0,1)+\Lambda(1,1))}{n}\right\} .
$$

ДокАЗАТЕльСтво. Обозначим $E=E_{n}^{r}(\Lambda)$. Для значений $E_{i}=i \cdot E, i=1,2, \ldots$, рассмотрим замкнутые множества

$$
W_{i}=(\Lambda)^{-1}\left(E_{i}\right) \cup\left\{z \in d: E_{i}-\frac{E}{4} \leqslant \Lambda(z) \leqslant E_{i}\right\} .
$$

Пусть $\left\{L_{j}\right\}_{j \in J}$ - те связные компоненты, составляюшие всевозможные $W_{i}$, которые не лежат целиком на $d$. Для каждого $j \in J$ обозначим через $i=i(j)$ индекс, при котором пересечение $L_{j} \cap(\Lambda)^{-1}\left(E_{i}\right) \neq \varnothing$. 
ЛЕмма 1. Кажсдое из мнохеств $L_{j}, j \in J$, мохсет быть задано как график непрерывного отобрахсения $g_{j}(\cdot)$ некоторого отрезка

$$
\left[x_{1}, x_{2}\right]=\left[x_{1}(j), x_{2}(j)\right] \subseteq[0,1]
$$

а именно

$$
L_{j}=\left\{z=\left(x, g_{j}(x)\right) \mid x \in\left[x_{1}, x_{2}\right]\right\}
$$

при этом

$$
\begin{aligned}
& \text { если } j_{1}, j_{2} \in J, j_{1} \neq j_{2}, i\left(j_{1}\right)=i\left(j_{2}\right), \text { mo }\left[x_{1}\left(j_{1}\right), x_{2}\left(j_{1}\right)\right] \cap\left[x_{1}\left(j_{2}\right), x_{2}\left(j_{2}\right)\right]=\varnothing ; \\
& \text { почки }\left(x_{s}, g_{j}\left(x_{s}\right)\right), s=1,2, \text { принадлежсат } d_{2} \\
& \text { если } x_{1}>0, \text { то } \Lambda\left(x_{1}, 1\right)=E_{i(j)}-E / 4 \\
& \text { если } x_{2}<1, \text { то } \Lambda\left(x_{2}, 1\right)=E_{i(j)}-E / 4 .
\end{aligned}
$$

ДокАЗАтЕльСтво. Пусть $W=W_{i(j)}, P=\left.\operatorname{Pr}_{x}\right|_{W}$ - оператор проектирования на ось $X$, ограниченньй на множество $W, \Delta=P(W)$. Так как отображение $\Lambda$ строго монотонно по $y$, то из определения $W$ следует, что любой отрезок вида $\{(x, y): y \in[0,1]\}$ пересекается с $W$ не более чем по одной точке, т.е. непрерьвное отображение $P$ является биекцией между компактом $W$ и $\Delta$, а значит, и их гомеоморфизмом. Отсюда и из того, что при гомеоморфизмах связные компоненты переходят в связные компоненты, следуют первые два утверждения леммы. Проверим третье. Пусть нам задана какая-нибудь связная компонента $\left[x_{1}, x_{2}\right]$ множества $\Delta$. Предположим, точка $\left(x_{1}, P^{-1}\left(x_{1}\right)\right) \notin d_{0} \cup d$. Тогда

$$
x_{1}>0, \quad 0<P^{-1}\left(x_{1}\right)<1 .
$$

Из свойств $\Lambda$ вытекает, что для некоторого малого $\delta>0$

$$
0=\Lambda(x, 0)<E_{i(j)}=\Lambda\left(x_{1}, P^{-1}\left(x_{1}\right)\right)<\Lambda(x, 1), \quad x \in\left[x_{1}-\delta, x_{1}\right],
$$

- противоречие со свойством максимальности компоненты. Аналогично показывается, что $\left(x_{2}, P^{-1}\left(x_{2}\right)\right) \in d_{1} \cup d$.

Пусть теперь $x_{1}>0$. Могут представиться два случая:

$$
\Lambda\left(x_{1}, 1\right)=E_{i(j)}-\frac{E}{4} \quad \text { или } \quad \Lambda\left(x_{1}, 1\right)=E_{i(j)} .
$$

Предположим, что $\Lambda\left(x_{1}, 1\right)=E_{i(j)}$. Выберем $\delta>0$ столь малым, чтобы $\Lambda(x, 1)>$ $E_{i(j)}-E / 4$ при $x \in\left(x_{1}-\delta, x_{1}\right)$. Тогда отрезок $\left[x_{1}-\delta, x_{2}\right]$ обязан принадлежать $\Delta$, а это противоречит условию, что $\left[x_{1}, x_{2}\right]-$ связная компонента $\Delta$. Утверждение, связанное с $x_{2}$, рассматривается аналогично. Лемма доказана.

Фиксируем $0<\varepsilon<E / 4$ (в дальнейшем мы устремим $\varepsilon$ к $E / 4)$. Пользуясь леммой 1 , для $j \in J$ по индукции определим последовательность $\left\{\tau_{k}\right\} \subset\left[x_{1}(j), x_{2}(j)\right]$ и функцию $s_{j}(\cdot)$ из $S_{k(j)}^{r}[0,1]$ следующим образом:

1) пусть $\tau_{0}=x_{1}(j), s_{j}\left(\tau_{0}\right)=g_{j}\left(\tau_{0}\right)$;

2 ) пусть $\tau_{k}$ определено и не совпадает с $x_{2}(j)$; тогда, если для некоторого $w$ такого, что $\left|E_{i(j)}-E / 2-w\right| \leqslant \varepsilon$, вьполняется

в случае а) $e\left(\tau_{k}, x_{2}(j), w\right) \leqslant \varepsilon$, в случае б) $\left.\left|w-\Lambda\left(x, p\left(x, \tau_{k}, x_{2}(j), w\right)\right)\right| \leqslant \varepsilon, p\left(x, \tau_{k}, x_{2}(j), w\right)\right) \in[0,1], x \in\left[\tau_{k}\right.$, $\left.x_{2}(j)\right]$, 
то $s_{j}(\cdot)=p\left(\cdot, \tau_{k}, x_{2}(j), w\right)$ на интервале $\left(\tau_{k}, x_{2}(j)\right), \tau_{k+1}=x_{2}(j), s_{j}\left(\tau_{k+1}\right)=g_{j}\left(\tau_{k+1}\right)$; если же это условие не выполняется, то

$$
\tau_{k+1}=\sup _{w:\left|E_{i(j)}-E / 2-w\right| \leqslant \varepsilon} \inf \left\{x \in\left(\tau_{k}, x_{2}(j)\right] \mid e\left(\tau_{k}, x, w\right) \geqslant \varepsilon\right\}
$$

и $s_{j}=p\left(\cdot, \tau_{k}, \tau_{k+1}, w\right)$ на интервале $\left(\tau_{k}, \tau_{k+1}\right]$ с параметром $w$, для которого sup в определении $\tau_{k+1}$ достигается.

Выберем $\sigma>0$ так, чтобы $\left|\Lambda\left(z_{1}\right)-\Lambda\left(z_{2}\right)\right|<\varepsilon$, если $\left|z_{1}-z_{2}\right|<\sigma$, и обозначим $w_{0}=E_{i(j)}-E / 2$. Тогда в случае а) определения последовательности $\tau_{k}$ величины $\tau_{k}$ и $\tau_{k+1} \neq x_{2}(j)$ не могут быть слишком близки:

$$
\begin{aligned}
\tau_{k+1} & \geqslant \inf \left\{x \in\left(\tau_{k}, x_{2}(j)\right]\left|\max _{t \in\left[\tau_{k}, x\right]}\right| w_{0}-\Lambda\left(t, h\left(\tau_{k}, w_{0}\right)\right) \mid \geqslant \varepsilon\right\} \\
& =\inf \left\{x \in\left(\tau_{k}, x_{2}(j)\right]\left|\max _{t \in\left[\tau_{k}, x\right]}\right| \Lambda\left(\tau_{k}, h\left(\tau_{k}, w_{0}\right)\right)-\Lambda\left(t, h\left(\tau_{k}, w_{0}\right)\right) \mid \geqslant \varepsilon\right\} \geqslant \tau_{k}+\sigma .
\end{aligned}
$$

Это означает, что процесс построения $\tau_{k}$ должен остановиться: $\tau_{k(j)}=x_{2}(j)$ для некоторого $k(j) \in \mathbb{N}$.

Предположим, что в случае б) определения последовательности $\tau_{k}$ процесс построения продолжается неограниченно. Тогда, поскольку $\Lambda(x, 0)=0, \Lambda(x, 1) \geqslant E_{i(j)}-E / 4$, $\Lambda\left(x, h\left(x, w_{0}\right)\right)=w_{0}=E_{i(j)}-E / 2$ при всех $x \in\left[x_{1}(j), x_{2}(j)\right]$ и

$$
\begin{aligned}
\tau_{k+1} & \geqslant \inf \left\{x \in\left(\tau_{k}, x_{2}(j)\right]|| w_{0}-\Lambda\left(x, p\left(x, \tau_{k}, w_{0}\right)\right) \mid \geqslant \varepsilon\right\} \\
& =\inf \left\{x \in\left(\tau_{k}, x_{2}(j)\right]|| \Lambda\left(\tau_{k}, p\left(\tau_{k}, \tau_{k}, w_{0}\right)\right)-\Lambda\left(x, p\left(x, \tau_{k}, w_{0}\right)\right) \mid \geqslant \varepsilon\right\},
\end{aligned}
$$

получим, что для каждого $k \in \mathbb{N}$ найдется точка $x^{*} \in\left(\tau_{k}, \tau_{k+1}\right]$ со свойством: расстояние между точками $\left(\tau_{k}, p\left(\tau_{k}, \tau_{k}, w_{0}\right)\right)$ и $\left(x^{*}, p\left(x^{*}, \tau_{k}, w_{0}\right)\right)$ на плоскости больше фиксированного $\sigma>0$. Это означает, что система коэффициентов $\left\{h_{x}^{(i)}\left(\tau_{k}, w_{0}\right)\right\}_{i=1}^{r}$ полинома $p\left(\cdot, \tau_{k}, w_{0}\right)$ не может оставаться ограниченной с ростом $k$, что противоречит условию $h(\cdot) \in C^{r}(H)$. Поэтому в случае б) процесс построения последовательности $\tau_{k}$ также обрывается: $\tau_{k(j)}=x_{2}(j)$ для некоторого $k(j) \in \mathbb{N}$.

Перечислим простые следствия сделанных построений:

1) функция $s_{j}(\cdot) \in S_{k(j)}^{r}[0,1]$ определена на $\left[x_{1}(j), x_{2}(j)\right]$;

2) $L^{j}=\bar{s}_{j}-$ связная линия без самопересечений, ее граничные точки $-\left(\tau_{0}, g_{j}\left(\tau_{0}\right)\right)$ и $\left(\tau_{k(j)}, g_{j}\left(\tau_{k(j)}\right)\right)$ расположены на $d_{2}$;

3) для любого $x_{0} \in\left[x_{1}(j), x_{2}(j)\right]$ множество $\left\{z \in L^{j} \mid z=\left(x_{0}, y\right)\right\}$ является точкой или отрезком;

4) для любого $z \in \bar{s}_{j}$

$$
\left|E_{i(j)}-\frac{E}{2}-\Lambda(z)\right| \leqslant 2 \varepsilon .
$$

Построим пары $g_{j}, L^{j}$ для всех $j \in J$. Из определения следует, что для любого $i$ и любого $w$ такого, что $\left|E_{i}-E / 2-w\right|<\varepsilon$, выполнена оценка

$$
\begin{gathered}
\Omega(w) \geqslant \sum_{j: i=i(j)}(k(j)-1) \cdot \varepsilon^{1 /(r+1)}, \\
\int_{\left[E_{i-1}, E_{i}\right]} \Omega(w) d w \geqslant 2 \sum_{j: i=i(j)}(k(j)-1) \cdot \varepsilon^{(r+2) /(r+1)} .
\end{gathered}
$$


Лемма 2. Если $j, k \in J, j \neq k$, mo $L^{j} \cap L^{k}=\varnothing$.

ДоКАЗАТЕЛЬСТВо. Если $i(j)=i(k)$, то по лемме 1

$$
\left[x_{1}(j), x_{2}(j)\right] \cap\left[x_{1}(k), x_{2}(k)\right]=\varnothing .
$$

По свойству 1)

$$
\operatorname{Pr}_{x}\left\{L^{j}\right\}=\left[x_{1}(j), x_{2}(j)\right]
$$

и аналогично для $k$, что доказьвает лемму в этом случае. Пусть $i(j)<i(k)$, и предположим, что $z \in L^{j} \cap L^{k}$. По свойству 4 )

$$
\left|E_{i(j)}-\frac{E}{2}-\Lambda(z)\right| \leqslant 2 \varepsilon, \quad\left|E_{i(k)}-\frac{E}{2}-\Lambda(z)\right| \leqslant 2 \varepsilon .
$$

Поэтому

$$
E_{i(k)}-E_{i(j)}=\left(E_{i(k)}-\frac{E}{2}-\Lambda(z)\right)-\left(E_{i(j)}-\frac{E}{2}-\Lambda(z)\right)<E
$$

- противоречие. Лемма доказана.

Обозначим через $|J|$ количество элементов в $J$.

Лемма 3. Имеет место оиенка

$$
\operatorname{Var}_{0}^{1} \Lambda(\cdot, 1)+\Lambda(0,1)+\Lambda(1,1) \geqslant|J| \cdot \frac{E}{2}
$$

ДокАЗАтЕЛЬСтво. Пусть $J_{0} \subset J$-множествовсех тех $j \in J$, для которых $x_{1}(j)=0$. Если для всех $j \in J_{0}$ вьполнено равенство $g_{j}(0)=E_{i(j)}$, то $\Lambda(0,1) \geqslant E_{\left|J_{0}\right|}=\left|J_{0}\right| \cdot E$; положим $x^{1}=0, \Delta=\left[0, x^{1}\right], \operatorname{Var}_{\Delta} \Lambda(\cdot, 1)=0$. Тогда

$$
\Lambda(0,1)+\operatorname{Var}_{\Delta} \Lambda(\cdot, 1) \geqslant \Lambda\left(x^{1}, 1\right) \geqslant\left|J_{0}\right| \cdot E .
$$

Если для некоторого $j \in J_{0}$ вьполнено $g_{j}(0)<E_{i(j)}$, то из определения $L_{j}$ следует, что $g_{j}(0)=1, i(j)=\left|J_{0}\right|$; положим $x^{1}=\min \left\{x \in[0,1] \mid \Lambda(x, 1)=E_{i(j)}\right\}$, $\Delta=\Delta_{1}(j)=\left[0, x^{1}\right]$. Тогда

$$
\{\Delta, 1\} \subset L_{j}
$$

и (3) также вьполнено. Допустим теперь, что $j \notin J_{0}$. По лемме 1

$$
g_{j}\left(x_{1}(j)\right)=1, \quad \Lambda\left(x_{1}(j), 1\right)=E_{i(j)}-\frac{E}{4}
$$

для $x^{1}(j)=\min \left\{x \in\left[x_{1}(j), 1\right] \mid \Lambda(x, 1)=E_{i(j)}\right\}, \Delta_{1}(j)=\left[x_{1}(j), x^{1}(j)\right]$ выполняется $(4)$ И

$$
\operatorname{Var}_{\Delta_{1}(j)} \Lambda(\cdot, 1) \geqslant \frac{E}{4} \text {. }
$$


По аналогии определяются $J_{1} \subset J$-множество всех тех $j \in J$, для которых $x_{2}(j)=1, x^{2}$,

$$
x^{2}(j)=\max \left\{x \in\left[0, x_{2}(j)\right] \mid \Lambda(x, 1)=E_{i(j)}\right\}, \quad \Delta_{2}(j)=\left[x^{2}(j), x_{2}(j)\right],
$$

удовлетворяющие (4), и разбираются неравенства

$$
\begin{gathered}
\Lambda(1,1)+\operatorname{Var}_{\Delta} \Lambda(\cdot, 1) \geqslant\left|J_{1}\right| \cdot E, \\
\operatorname{Var}_{\Delta_{2}(j)} \Lambda(\cdot, 1) \geqslant \frac{E}{4} .
\end{gathered}
$$

Согласно определению и условию (4) никакие два разньг отрезка из набора $\left\{\Delta_{1}(j)\right.$, $\left.\Delta_{2}(j)\right\}, j \in J$, не пересекаются. Поэтому

$$
\operatorname{Var}_{0}^{1} \Lambda(\cdot, 1) \geqslant \sum_{j}\left(\operatorname{Var}_{\Delta_{1}(j)} \Lambda(\cdot, 1)+\operatorname{Var}_{\Delta_{2}(j)} \Lambda(\cdot, 1)\right)
$$

(в сумме справа любое из неопределенных слагаемых считается равным нулю). Совмещая последнее неравенство с (3), (6), (5), (7), получим утверждение леммы.

Ниже мы покажем, что

$$
n \leqslant \sum_{j \in J}(k(j)+1)
$$

а сейчас, пользуясь этим фактом, установим оценку теоремы: в силу (8) выполнено хотя бы одно из двух условий:

1) $\sum_{j \in J}(k(j)-1) \geqslant n / 2$ или

2) $|J| \geqslant n / 4$.

Поэтому либо по лемме 3

$$
E_{n}=E \leqslant \frac{8(I(\Lambda)+\Lambda(0,1)+\Lambda(1,1))}{n},
$$

либо по (2)

$$
(\varepsilon)^{(r+2) /(r+1)} \leqslant \frac{I(\Lambda, r)}{n} .
$$

Поскольку $\varepsilon<E / 4$ может быть выбрано сколь угодно близким к $E / 4$, из этих неравенств следует утверждение теоремы. Таким образом остается проверить (8). Для этого с помошью введенных функций $g_{j}$ построим аппроксимацию $\Lambda$.

Множество $L^{j}$ разбивает квадрат I на некоторое количество связных открытых в I компонент. Согласно свойству 4) $L^{j}$ не пересекается с отрезком

$$
d_{3}=\{z=(x, 0) \mid x \in[0,1]\} .
$$

Поэтому одна из компонент - обозначим ее $U_{j}$ - содержит этот отрезок.

Лемма 4. Пусть $j \in J, x \in[0,1]$. Тогда для $\Delta(x)=\{z=(x, y) \mid y \in[0,1]\}$ выполнены утверждения:

1) $\Delta(x) \subset U_{j}$ nрu $x \notin\left[x_{1}(j), x_{2}(j)\right]$;

2) $\Delta(x) \cap U_{j}=:\{z=(x, y) \mid y \in[0, y(x))\}, \quad(x, y(x)) \in L^{j}$, npu $x \in\left[x_{1}(j), x_{2}(j)\right]$ $\left(\right.$ здесь $\left.y(x)=y_{j}(x)\right)$. 
ДоКАЗАТЕЛЬСТВО этой леммы прямо следует из определений.

Зададим функцию $F$ на I следующим образом: пусть $z \in \mathbf{I}$, тогда

$$
F(z)= \begin{cases}0, & \text { если } z \in U_{j} \text { при всех } j \in J, \\ \max \left\{E_{i(j)} \mid z \notin U_{j}, j \in J\right\} & \text { в остальных случаях. }\end{cases}
$$

Лемма 5. Функиия $F$ принадлежит классу $S_{N}^{r}$ при некотором $N \in \mathbb{N}$. При этом точки разрыва $F$ лежат на $L^{k}, k \in J$, а линии разрыва состоят из звеньев, образующих $L^{k}, k \in J$.

ДокАЗАТЕльСтво. Как следует из построения, $F$ определена на I и принимает конечное число значений. Пусть $x \in[0,1], 0<y_{1}<y_{2} \leqslant 1$. По лемме 4 , если $y_{1} \notin U_{j}$ при каком-нибудь $j \in J$, то и $y_{2} \notin U_{j}$. Поэтому $F$ удовлетворяет условию монотонности из определения 1. Обозначим

$$
I^{\prime}=\mathbf{I} \backslash\left\{\bigcup_{j \in J} L^{j}\right\}
$$

открытоев I множество. Из свойства монотонности и того, что $F(z) \in F\left(I^{\prime}\right)$ для $z \in L^{j}$, следует условие 3 ) определения 2 . Пусть $M$ - какая-нибудь связная компонента $I^{\prime}$. Так как $M$ не пересекается ни с одним $L^{j}, j \in J$, то для каждого $j \in J$ либо $M \subseteq U_{j}$, либо $M \cap U_{j}=\varnothing$. Поэтому $F$ постоянна на $M$ и, значит, кусочно-постоянна на I. В силу открытости $I^{\prime}$ в I отсюда следует также, что $F$ непрерьвна во всех точках $I^{\prime}$. Это доказьвает второе утверждение леммы.

Проверим последнее. По определению гранища $M$ в I лежит в $\bigcup_{j \in J} L^{j}$; пусть $\partial-$ ее связная компонента. По лемме 2 для некоторого $j \in J$ выполнено включение $\partial \subseteq L^{j}$. По свойству 3) $L^{j}$ гомеоморфно отрезку, поэтому $\partial$ также гомеоморфно отрезку и определены $z_{1}, z_{2}-$ концы $\partial$. Далее, предположим $z_{1}$ (или $z_{2}$ ) лежит внутри некоторого $\Delta$, являющегося звеном $L^{j}$ из (1):

$$
z_{1} \in \Delta=\left\{\left(a_{i}, y\right) \mid y \in\left(p_{i-1}\left(a_{i}\right), p_{i}\left(a_{i}\right)\right)\right\} \quad \text { или } \quad \Delta=\left\{(x, s(x)) \mid x \in\left(a_{i}, a_{i+1}\right)\right\}
$$

при некотором $i$. Выберем малую окрестность $U \subset \mathbf{I}$ точки $z_{1}$ (круг, если $z_{1} \notin d_{2}$, или половину круга, если $z_{1} \in d_{2},-$ по предположениям $z_{1}$ не является угловой точкой $\left.\mathbf{I}\right)$, которая из всех звеньев, составляющих $L^{k}$ при всех $k \in J$, пересекается только с $\Delta$. При необходимости уменьшая $U$, можно считать, что $\Delta$ разбивает $U$ на не более чем две части, одна из которых лежит в $M$. Однако это противоречит определению $z_{1}$ как граничной точки $\partial$. Таким образом, линии разрьва $F$ состоят из частей, которые являются звеньями $L^{k}, k \in J$. Аналогично показывается, что $z_{1}$ (как и $z_{2}$ ) принадлежат $d_{2}$.

Отметим, таким образом, что гранища $M \backslash d_{2}$ состоит либо из линий разрыва $F$, либо отрезков на $d_{2}$. Поэтому, так как замыкание каж дой области постоянства $F$ совпадает с замыканием некоторого множества $\bigcup M_{j}$, где $M_{j}$ - множества типа $M$, а индекс $j$ пробегает некоторьй специфичньй для данной области набор индексов из $J$, то условие 2 ) определения 1 , а вместе с ним и лемма доказаны.

ЛЕмма 6. Имеет место оиенка

$$
\|\Lambda-F\|_{C(I)} \leqslant E .
$$


ДокаЗАТЕЛьство. По лемме 5 , определению класса $S_{N}^{r}$ и непрерывности $\Lambda$ на $\mathbf{I}$ требуемое для доказательства (9) неравенство

$$
|\Lambda(z)-F(z)| \leqslant E
$$

достаточно проверить для $z=(x, y) \in \mathbf{I}, z \notin d_{2}, z \notin L^{j}$ при всех $j \in J$.

Если $z \in U_{j}$ при всех $j \in J$, то

$$
0 \leqslant \Lambda(z) \leqslant E .
$$

Действительно, пусть $\Lambda(z)>E$. Тогда для некоторого $j \in J, i(j)=1$, множество $L_{j}$ отделяет $z$ от $d_{3}$ на $\mathbf{I}$. В силу леммы $1 x \in\left[x_{1}(j), x_{2}(j)\right]$. Пользуясь леммой 4 и свойством 4), получим

$$
y<y_{j}(x), \quad \Lambda(x, y)<\Lambda\left(x, y_{j}(x)\right) \leqslant E_{i(j)}=E
$$

- противоречие. Следовательно, для таких $z$

$$
|\Lambda(z)-F(z)|=|\Lambda(z)| \leqslant E .
$$

Пусть $z \notin U_{j}, j \in J$; тогда $F(z) \geqslant E_{i(j)}$; предположим, что $\Lambda(z) \leqslant(i(j)-1) E$. По лемме $4 x \in\left[x_{1}(j), x_{2}(j)\right]$, по свойству 4 )

$$
\Lambda(z) \leqslant(i(j)-1) E<\Lambda\left(x, y_{j}(x)\right),
$$

т.е. $0 \leqslant y<y_{j}(x), L^{j}$ не отделяет $z$ от $d_{3}, z \in U_{j}$ - противоречие. Поэтому

$$
\Lambda(z)>(i(j)-1) E, \quad \Lambda(z)-E_{i(j)}>-E .
$$

Выберем $j \in J$ так, чтобы $z \notin U_{j}$ и чтобы $F(z)=E_{i(j)}$. Тогда

$$
\Lambda(z)-F(z) \geqslant-E .
$$

Предположим $\Lambda(z)>(i(j)+1) E$ и, значит, найдется $j_{0} \in J, i\left(j_{0}\right)=(i(j)+1)$, такое, что множество $L_{j_{0}}$ отделяет $z$ от $d_{3}$ на $\mathbf{I} ;$ по лемме $1 x \in\left[x_{1}\left(j_{0}\right), x_{2}\left(j_{0}\right)\right]$. В силу определения индекса $i(j)$ точка $z \in U_{j_{0}}$. По лемме 4 и свойству 4 )

$$
\Lambda(x, y)<\Lambda\left(x, y_{j_{0}}(x)\right) \leqslant E_{i\left(j_{0}\right)}
$$

- противоречие. Таким образом, $\Lambda(z)-F(z) \leqslant E$. Комбинируя это неравенство с (11), получаем (10). Лемма доказана.

Согласно леммам 5 и $6 F \in S_{N}^{r}, E_{N} \leqslant E=E_{n}$, а значит, $n \leqslant N \leqslant \sum_{j \in J}(k(j)+1)$. Неравенство (8), а вместе с ним и теорема доказаны.

Пусть $s_{n}$ - класс кусочно-постоянных на $[0,1]$ функций с числом интервалов постоянства не выше $n \in \mathbb{N}$ со значениями в $\mathbb{R}$. 
СлЕДСТВИЕ. Пусть $r \in \mathbb{Z}_{+}, n \in \mathbb{N}$. Для любого $\varepsilon>0$ найдутся функиии $F_{1}, F_{2} \in S_{n}^{r}, F_{3}(x, y) \equiv s(x), s(\cdot) \in s_{n}$, такие, что

$$
\begin{aligned}
& \left\|f-F_{1}+F_{2}-F_{3}\right\|_{B} \leqslant 4 \max \left\{\left(\frac{I(\Lambda, r)}{n}\right)^{(r+1) /(r+2)}, \frac{2(I(\Lambda)+\Lambda(0,1)+\Lambda(1,1))}{n}\right\} \\
& +4 \max \left\{\left(\frac{I(\mathscr{M}, r)}{n}\right)^{(r+1) /(r+2)}, \frac{2(I(\mathscr{M})+\mathscr{M}(0,1)+\mathscr{M}(1,1))}{n}\right\}+\frac{\operatorname{Var}_{0}^{1}(f(\cdot, 0))}{n}+\varepsilon .
\end{aligned}
$$

ДокАЗАТЕЛЬСТвО этого утверждения следует из теоремы, разложения $f(x, y)=$ $\Lambda(x, y)-\mathscr{M}(x, y)+f(x, 0),(x, y) \in \mathbf{I}$, и хорошо известного неравенства $e_{n}(f(\cdot, 0)) \leqslant$ $\operatorname{Var}_{0}^{1}(f(\cdot, 0)) / n$ (здесь $e_{n}(f(\cdot, 0))$ - величина наилучшего приближения функциями из $s_{n}$ в метрике пространства $B$ на отрезке $[0,1])$.

ЗАМЕчАНИЕ 3 . В случае $r=0$ вместо $I(\Lambda, 0)$ можно использовать величину

$$
I^{\star}(\Lambda, 0)=\sup _{\substack{u(\cdot) \in C_{0}^{1}\left((0,1)^{2}\right) \\\|u\|_{C} \leqslant 1}} \int_{0}^{\lambda} \omega \cdot\left\{\int_{z \in L_{\omega}} \frac{\partial u}{\partial x}(z) d x\right\} d \omega
$$

(здесь $L_{\omega}=\{z \in \mathbf{I} \mid \Lambda(z)=\omega\}, C_{0}^{1}\left((0,1)^{2}\right) \subset C^{1}(\mathbf{I})-$ класс гладких функций с носителем внутри $\left.(0,1)^{2}\right)$ и незначительным изменением деталей доказательства установить справедливость теоремы и следствия, когда вместо $I(\Lambda, 0), I(\mathscr{M}, 0)$ стоят $I^{\star}(\Lambda, 0)$, $I^{\star}(\mathscr{M}, 0)$.

\section{СПИСОК ЦИТИРОВАННОЙ ЛИТЕРАТУРЫ}

[1] Kochurov A. S. Approximation by piecewise constant functions on the square // East J. Approximations. 1995. V. 1. № 4. P. 463-478.

Московский государственный университет

Поступило им. М. В. Ломоносова

25.07 .2000

E-mail : kochurov@math.msu.su 